Submission ID: 43711

\title{
Use of Modelling in the Interpretation of DNME Data to the Improving
} Forecast Accuracy

S.J. Garina* (Siberian Geophysical Research Production Company LLC), S.A. Ivanov (Siberian Geophysical Research Production Company LLC), V.V. Vladimirov (Siberian Geophysical Research Production Company LLC), I.Yu. Pesterev (Siberian Geophysical Research Production Company LLC)

\section{SUMMARY}

In DNME, the estimation parameters are the polarization parameters and electrical resistivity. As is well known, the IP anomalies can be associated not only with the presence of $\mathrm{HC}$, but also with lithological changes and three-dimensional heterogeneities in the overburden. We also know about the ambiguity of the inverse problem solution. The use of modelling of various geological situations works towards a solution of controversial questions about the connection of the allocated polarization objects with $\mathrm{HC}$ or another source. For this purpose GeoEM software (development of M.G. Persova) is designed to solve three-dimensional geoelectric exploration problems, as well as a program ESDesigner for modeling of a deeper sectional streamer position and calculating of direct problem (development of I.Yu. Pesterev) are currently used in the SGRPC LLC. The results obtained from the synthetic models in comparison with the results of inversion and a priori data made it possible to take into account the degree of influence of the large water layer on the DNME parameters and to select target polarizing layers. It is shown that the vertical zonality of the IP fields' positions in the section and the polarizable layers corresponding to them can be reversed. 
Использование моделирования в ходе интерпретации данных ДНМЭ для повышения надежности прогноза

С.Ю. Гарина* (ООО «СГНПК»), С.А. Иванов (ООО «СГНПК»), В.В. Владимиров (ООО «СГНПК»), И.Ю. Пестерев (ООО «СГНПК»)

\section{Введение}

Работы ДНМЭ проводятся в самых разных геологических условиях на суше и на море. Геоэлектрическими параметрами оценки являются поляризационные параметры (коэффициент поляризуемости $\eta$, поле поляризуемости IPg, время релаксации $\tau$ ) в совокупности с удельным электрическим сопротивлением. Данный метод позволяет устанавливать наличие скоплений УВ в ловушках как структурного, так и неструктурного типа. При этом, как известно, аномалии ВП могут быть связаны не только с наличием УВ, но и с литологическими изменениями и трехмерными неоднородностями в ВЧР. С другой стороны, также известно о неоднозначности решения обратной задачи.

Решению спорных вопросов о связи выделенных поляризационных объектов с теми или иными причинами способствует использование моделирования различных геологических ситуаций с последующей инверсией полученных синтетических данных. В ООО «СГНПК» для этих целей на данный момент используется программный комплекс GeoEM (разработка М.Г. Персовой, ООО «Техпроект», г. Новосибирск), разработанный для решения трехмерных задач геоэлектроразведки и позволяющий моделировать геоэлектромагнитные процессы в горизонтально-слоистой вмещающей среде с трехмерными объектами, а также программа моделирования положения погружной секционной косы и расчета прямой задачи ESDesigner (разработка И.Ю. Пестерева, ООО «СГНПК», г. Иркутск).

Моделирование с использованием указанных программ позволяет проводить ранжирование выделенных аномалий ВП и повысить надежность прогноза наличия УВ.

\section{Использование 1D и 3D моделирования в ДНМЭ}

Морские работы ДНМЭ проводятся в непрерывном цикле работ плавучей, либо погружной косой на глубинах до 1 км, включая транзитную зону. Разрез возбуждается прямоугольными разнополярными импульсами тока скважностью 2 периодом 32 с. Сигналы переходных процессов регистрируются во временном диапазоне наблюдений с 26 до 7500-12000 мс. С каждой трехэлектродной установки $\mathrm{X}_{\mathrm{A}} \mathrm{X}_{\mathrm{B}} \mathrm{X}_{\mathrm{C}}$ сигнал поступает в измерительный усилитель, где формируются две кривые спада $\left(\Delta^{2} U\right.$ и $\left.\Delta U\right)$ для каждого из трех разносов, которые затем подаются на АЦП. После АЦП данные поступают в компьютер, где обрабатываются программой «Сбора и сохранения массовых данных». Трансформанта D2U является, главным образом, характеристикой ВП. Инверсия данных осуществляется на основе модели Коул-Коул. Входными данными обратной задачи являются наблюденные полевые кривые $D U$ и трансформанты $P 1, P S, D \phi S$, получаемые на основе $D U$ и $D 2 U$ одновременно для всех трех разносов. Временной диапазон, используемый при моделировании, определялся достоверностью входных данных, т.е. точностью измерений каждого из параметров на каждом разносе отдельно.

Одновременная минимизация входных данных с разным соотношением ВП/электродинамика открывает путь к количественному разделению полей (Пат. 2399931, 2010). Технология ДНМЭ позволяет осуществлять количественное разделение процессов электромагнитной индукции (EM) и вызванной поляризации (ВП) в широком временном диапазоне. Однако практическое использование такого диапазона осложняется влиянием на получаемый сигнал изменениями глубины моря и сопротивления морской толщи.

Получаемый в ДНМЭ поляризационный отклик является совокупным от всего интервала разреза. Для выделения разноуровневых залежей необходимо привлечение дополнительных 
данных по другим методам, в первую очередь - по сейсморазведке. Интерпретация осложняется неизбежным при решении обратной задачи появлением эквивалентных зависимостей между геоэлектрическими параметрами.

Проведение моделирования в программах GeoEM и ESDesigner с последующей инверсией полученных синтетических данных позволяет найти основу для ранжирования аномалий ВП, обусловленных различными геологическими объектами.

Использование простой модели разреза с выдержанными мощностями и присутствием поляризующегося объекта в ВЧР (на глубинах до 1 км) позволяет зафиксировать наличие объекта по трансформанте D2U во всем временном интервале как в программе ESDesigner (Рис.1), так и в программном комплексе GeoEM (Рис. 2).

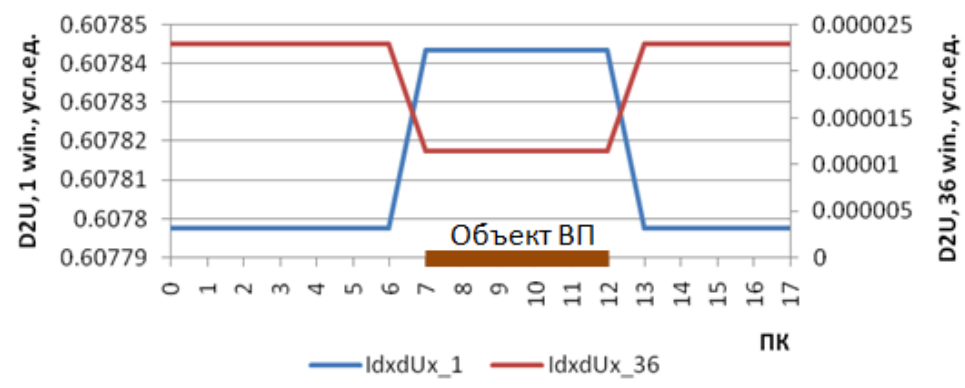

Рисунок 1 Изменение распределения D2U над объектом ВП на 1-м (0.027 c) и 36-м (12 c) временных окнах, полученное в программе ESDesigner.

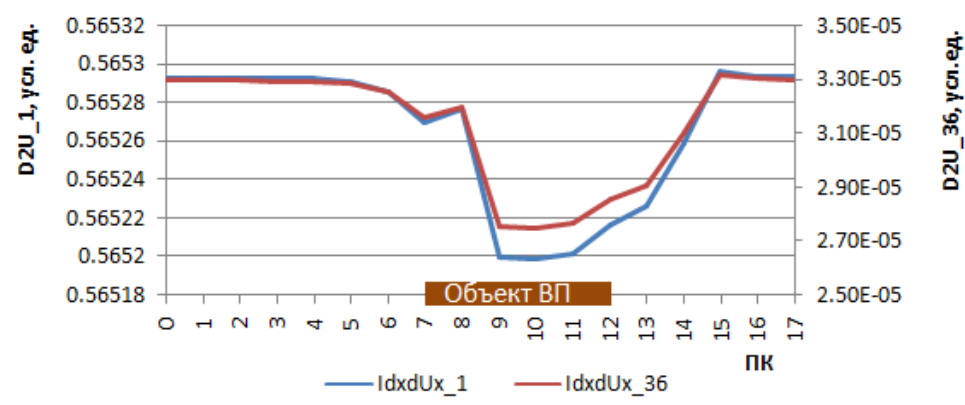

Рисунок 2 Изменение распределения D2U над объектом ВП на 1-м (0.027 с) и 36-м (12 c) временных окнах, полученное в программном комплексе GеоЕМ.

Различия в распределении трансформанты D2U на ранних временах обусловлены в различиях задаваемой геометрии косы в данных программах - в ESDesigner имеется возможность задать наклонное положение генераторной линии, в GeoEM такая возможность отсутствует.

При работах в морских условиях по реальным данным на большей части временного интервала обнаруживается существенное влияние на распределение D2U изменений глубин моря. Использование при моделировании изменяющихся глубин моря подтверждает затушевывающее полезный отклик влияние изменения глубин морского дна на ранних окнах как при значительных (от 300 до 1200 м), так и при небольших (640-700 м) изменениях глубин (Рис. 3,4$)$. На поздних временах спада (начиная с 34 временного окна, соответствующего 4 с времени спада) объект проявляется отчетливо. 


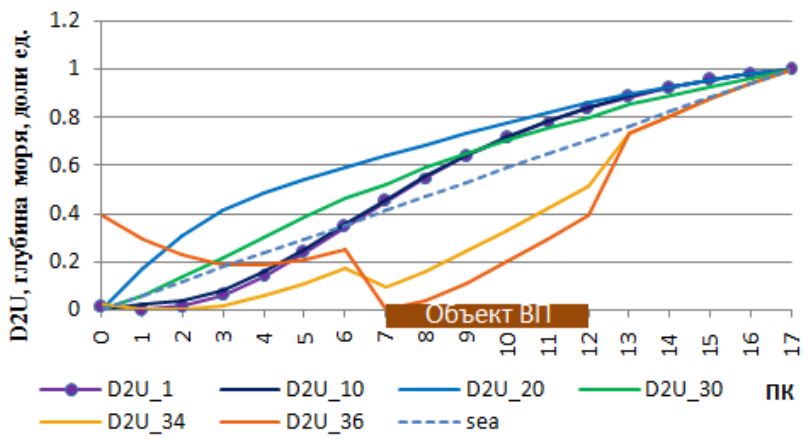

Рисунок 3 Изменение распределения D2U над объектом ВП на разных временных окнах в условиях колебания дна моря от 300 до 1200 м в сопоставлении с глубинами моря (все значения приведены в долях единищы).

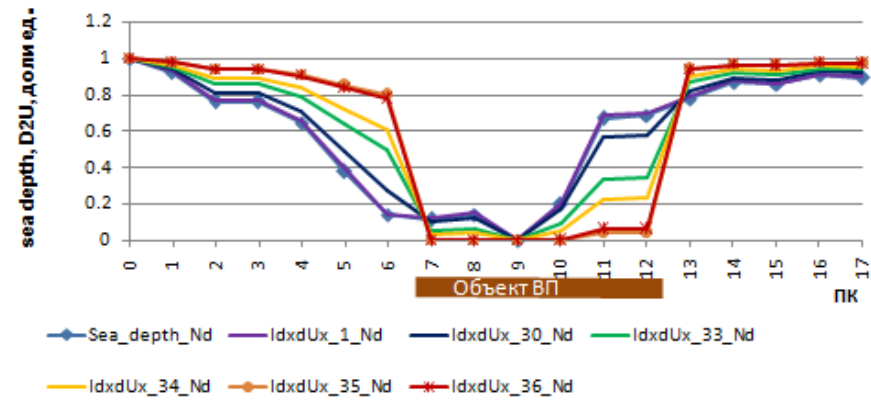

Рисунок 4 Изменение распределения D2U над объектом ВП на разных временных окнах в условиях колебания дна моря от 640 до 700 м в сопоставлении с глубинами моря (все значения приведены в долях единищы).

Инверсия 1D, проведенная для полученных в обеих программах синтетических данных, демонстрирует воспроизводимость геоэлектрического разреза на качественном уровне, при этом аномалия, полученная на основе 3D моделирования, несколько смещена по ходу судна и получена в том же слое, в котором задан объект ВП в модели, в то время как при использовании 1D моделирования аномалия получена выше (Рис. 5).

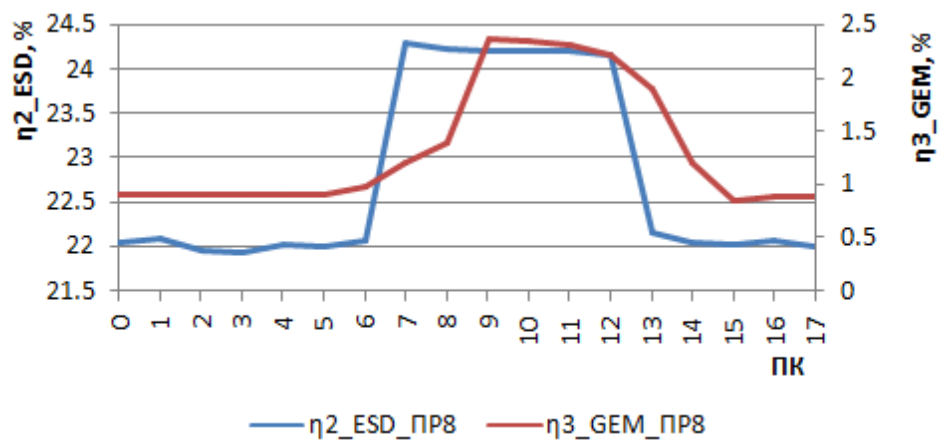

Рисунок 5 Распределение коэффициента поляризуемости $\eta$ по результатам инверсии данных моделирования с использованием программы ESDesigner u GeоEM при фиксированных глубинах моря.

Таким образом, 3D моделирование на данном этапе обеспечивает результат более близкий к реальности при простом строении разреза, включающего трехмерные объекты, в то время как программа ESDesigner позволяет моделировать более сложную геометрию среды. 


\section{Опыт 3D моделирования в относительно глубоководных условиях работ ДНМЭ}

При работах на глубинах около 1 км на черноморском шельфе при инверсии полевых данных был получен странный факт: аномалии ВП в более глубоком 4-м слое по геологическим данным увязывались с верхами разреза, в то время как более глубоко расположенные залежи УВ, подтвержденные бурением, отражались в аномалиях ВП, расположенных в более высоко расположенном 3-м слое. 3D моделирование показало принципиальную возможность таких результатов инверсии (Рис. 6), что обеспечило более надежное ранжирование полученных аномалий ВП.

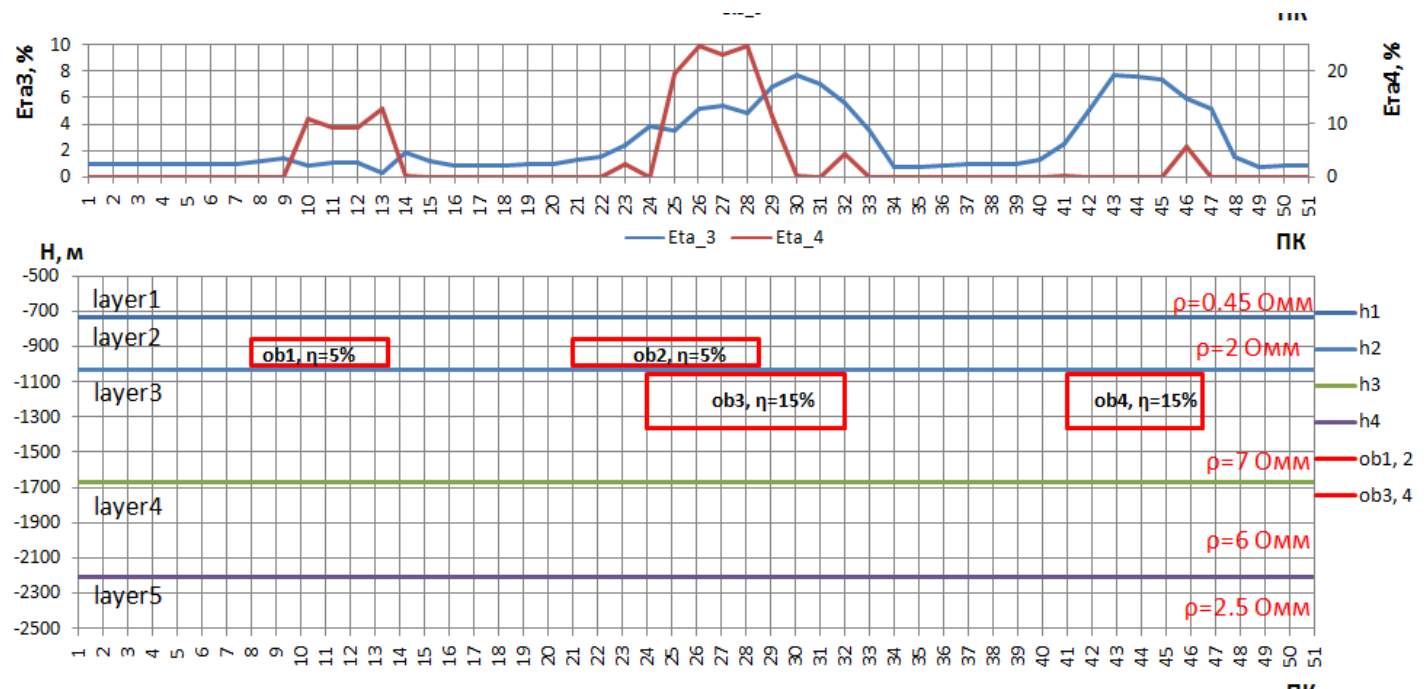

пк

Рисунок 6 Результаты 3D моделирования, демонстрирующие возможность отражения объектов ВП в ВЧР в более глубоко расположенных геоэлектрических слоях.

\section{Выводы}

Полученные по синтетическим моделям результаты в сопоставлении с результатами инверсии и априорными данными позволили учесть степень влияния мощного водного слоя на параметры ДНМЭ и выбрать целевые поляризующиеся слои, наиболее тесно связанные с вероятным расположением залежей в разных интервалах разреза. При этом показано, что вертикальная зональность расположения залежей в разрезе и отвечающих им поляризующихся слоев может быть обратной. Таким образом, моделирование является важным инструментом интерпретации данных ДНМЭ, позволяющим повысить надежность прогноза наличия УВ.

\section{Благодарности}

Выражаем глубокую признательность М.Г. Персовой за плодотворное сотрудничество. Выражаем благодарность ЛУКОЙЛ Оверсиз Аташ Б.В. (Филиал в г. Бухаресте) за предоставленные геологические материалы.

\section{Библиография}

1. Пат. 2399931 Российская Федерация, МПК G01V 3/38, G01V3/06. Способ количественного разделения эффектов электромагнитной индукции и вызванной поляризации [Текст] / Легейдо П.Ю., Иванов С.А., Давыденко Ю.А. и др. (РФ); заявитель и патентообладатель ООО «Сибирская геофиз. науч.-произв. комп-я». - № 2008141889, опубл. 20.09.2010; приоритет 23.10.2008. 


\section{EAGE}

\section{References}

1. Patent 2399931 Russian Federation, IPC G01V 3/38, G01V3/06. The method of quantitative separation of the effects of electromagnetic induction and induced polarization [Text] / Legeido P.Yu., Ivanov S.A, Davydenko Yu.A. and others (RF); Applicant and patent owner of LLC "Siberian Geophysical Research Production Company". - No. 2008141889, publ. 20.09.2010; Priority is on 23.10.2008. 